\title{
Editorial
}

\section{Advanced Time Series Forecasting Methods}

\author{
Erol Egrioglu, ${ }^{1}$ Mehdi Khashei, ${ }^{2}$ Cagdas Hakan Aladag, ${ }^{3}$ \\ I. Burhan Turksen, ${ }^{3}$ and Ufuk Yolcu ${ }^{4}$
}

${ }^{1}$ Department of Statistics, Faculty of Arts and Science, Marmara University, Göztepe Campus, Kadikoy, 34700 Istanbul, Turkey
${ }^{2}$ Department of Industrial Engineering, Faculty of Science, Isfahan University of Technology (IUT), Isfahan 84156-83111, Iran
${ }^{3}$ Knowledge/Intelligence Systems Laboratory, Department of Mechanical \& Industrial Engineering, University of Toronto,
Toronto, ON, Canada M5S $3 G 8$
${ }^{4}$ Department of Statistics, Faculty of Science, Ankara University, 06100 Ankara, Turkey

Correspondence should be addressed to Erol Egrioglu; erole1977@yahoo.com

Received 21 May 2015; Accepted 27 May 2015

Copyright (C) 2015 Erol Egrioglu et al. This is an open access article distributed under the Creative Commons Attribution License, which permits unrestricted use, distribution, and reproduction in any medium, provided the original work is properly cited.

In recent years, various computational and nonprobabilistic forecasting methods have been proposed in the literature. Since probabilistic forecasting methods have strict assumptions and probability is not a unique solution for every kind of uncertainty, contemporary forecasting methods have become popular. Contemporary forecasting methods are fuzzy theory based methods, computational methods like artificial neural networks, and hybrid methods which are different combination of probabilistic, fuzzy, and computational methods. These methods can also be called intelligent forecasting methods. Aim of this special issue was creating a reference work for intelligent forecasting methods. Brief information about the published studies can be found below.

P. Göktaş and C. Dişbudak from Turkey in "Modelling Inflation Uncertainty with Structural Breaks Case of Turkey (1994-2013)" employed both symmetric and asymmetric GARCH-type models to determine the best representative of the inflation uncertainty. The structural breaks and stationary situation were identified for Turkish inflation series and EGARCH $(1,1)$ model was used in the determination of inflation uncertainty. According to results of econometrical applications, some economic comments were put forward for the real world economic events of Turkish economy.

C. Kocak from Turkey in "A New High Order Fuzzy ARMA Time Series Forecasting Method by Using Neural Networks to Define Fuzzy Relations" proposed a new fuzzy time series method and this method employed high order fuzzy time series forecasting methods with lagged error variables. According to real world applications, it was proved that the new fuzzy time series methods can produce accurate forecasts from some other methods in the literature.

J. Yuan et al. from China in "Ultrahigh Frequency Data Liquidity Duration Estimation: A Case Study of Chinese A Shares" analyzed Asian emerging market equities liquidity using ultrahigh frequency data by using Weibull autoregressive conditional duration model, the best model to describe the overall liquidity.

$X$. Chen et al. from China in "Short-Term Wind Speed Forecasting Study and Its Application Using a Hybrid Model Optimized by Cuckoo Search" proposed a new hybrid SVR and cuckoo search optimization methods. The new hybrid method improved the performance of SVR method by using data preprocessing and cuckoo search optimization.

C. Xie et al. from China in "Forecasting RMB Exchange Rate Based on a Nonlinear Combination Model of ARFIMA, SVM, and BPNN" proposed a nonlinear combination model of the autoregressive fractionally integrated moving average (ARFIMA) model, the support vector machine (SVM) model, and the backpropagation neural network (BPNN) model to forecast the $\mathrm{RMB}$ exchange rate. The empirical results showed that the prediction performance of the nonlinear combination model is better than the single models.

C.-C. Young et al. from Taiwan in "Predicting the Water Level Fluctuation in an Alpine Lake Using Physically Based, Artificial Neural Network, and Time Series Forecasting Models" used hydrodynamic models, ARMAX, ANN, and 
combined hydrodynamic and ANN model to predict water level. As a result of the paper, it is clear that combined approach produced the best results.

An approach based on Sparse Bayesian Learning (SBL) and Numerical Weather Prediction (NWP) for probabilistic wind power forecasting in the horizon of 1-24 hours was investigated by K. Pan et al. from China in "Probabilistic Short-Term Wind Power Forecasting Using Sparse Bayesian Learning and NWP." To validate the proposed approach, two real world datasets were used for construction and testing and the proposed method produced the best results.

A novel local nonlinear model called local polynomial coefficient autoregressive prediction (LPP) model based on the phase space reconstruction was proposed by L. Su and C. Li from China in "Local Prediction of Chaotic Time Series Based on Polynomial Coefficient Autoregressive Model." As a result of this study, the LPP model can effectively fit nonlinear characteristics of chaotic time series with simple structure and have excellent one-step forecasting performance. Moreover, a kernel LPP (KLPP) model which applies the kernel technique for the LPP model to obtain better multistep forecasting performance was proposed. The proposed models are flexible to analyze complex and multivariate nonlinear structures.

Y. Lyu from China in "Detection of Outliers in Panel Data of Intervention Effects Model Based on Variance of Remainder Disturbance" presented a modeling method to assess the intervention effects based on the variance of remainder disturbance in order to detect outliers in panel data models.

J. Jin et al. from China in "Data Normalization to Accelerate Training for Linear Neural Net to Predict Tropical Cyclone Tracks" proposed a new data normalization strategy for pure linear neural network (PLNN) to predict tropical cyclone tracks (TCT). Some useful results were obtained for data normalization.

P. Jiang et al. from China in "Time Series Analysis and Forecasting for Wind Speeds Using Support Vector Regression Coupled with Artificial Intelligent Algorithms" proposed an intelligent hybrid model for short-term wind speed prediction. The proposed hybrid models were used to forecast short-term wind speeds collected from four wind turbines located on a wind farm in China and the intelligent hybrid models outperform some other methods in the literature.

A. Shabri and R. Samsudin from Malaysia in "Fishery Landing Forecasting Using Wavelet-Based Autoregressive Integrated Moving Average Models" proposed an ensemble approach based on ARIMA model and wavelet transform. The proposed model was found to provide more accurate fishery landing series forecasts than the individual ARIMA model.

The authors contributed to the time series literature with high quality papers. The special issue is very useful for forecast researchers. Different forecasting approaches were introduced in the special issue. Specifically, new hybrid and combined approaches were proposed in these papers. The special issue can be a good guide for potential probabilistic, nonprobabilistic, and hybrid forecasting approaches for forecast researchers in this area.

Erol Egrioglu

Mehdi Khashei

Cagdas Hakan Aladag

I. Burhan Turksen

Ufuk Yolcu 


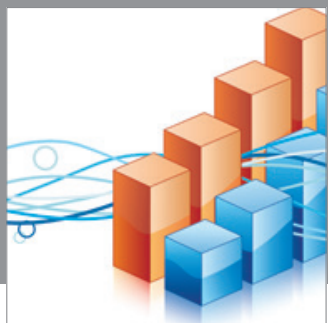

Advances in

Operations Research

mansans

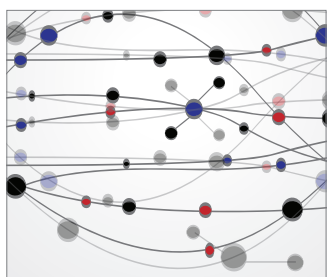

The Scientific World Journal
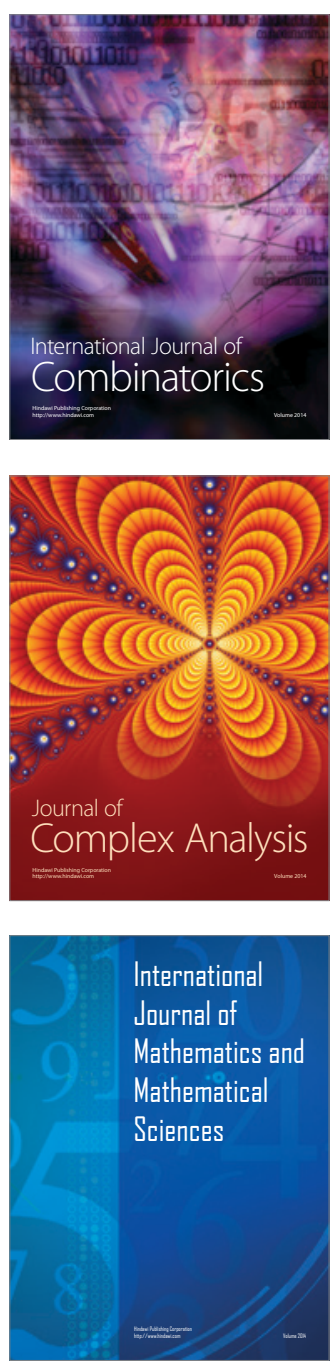
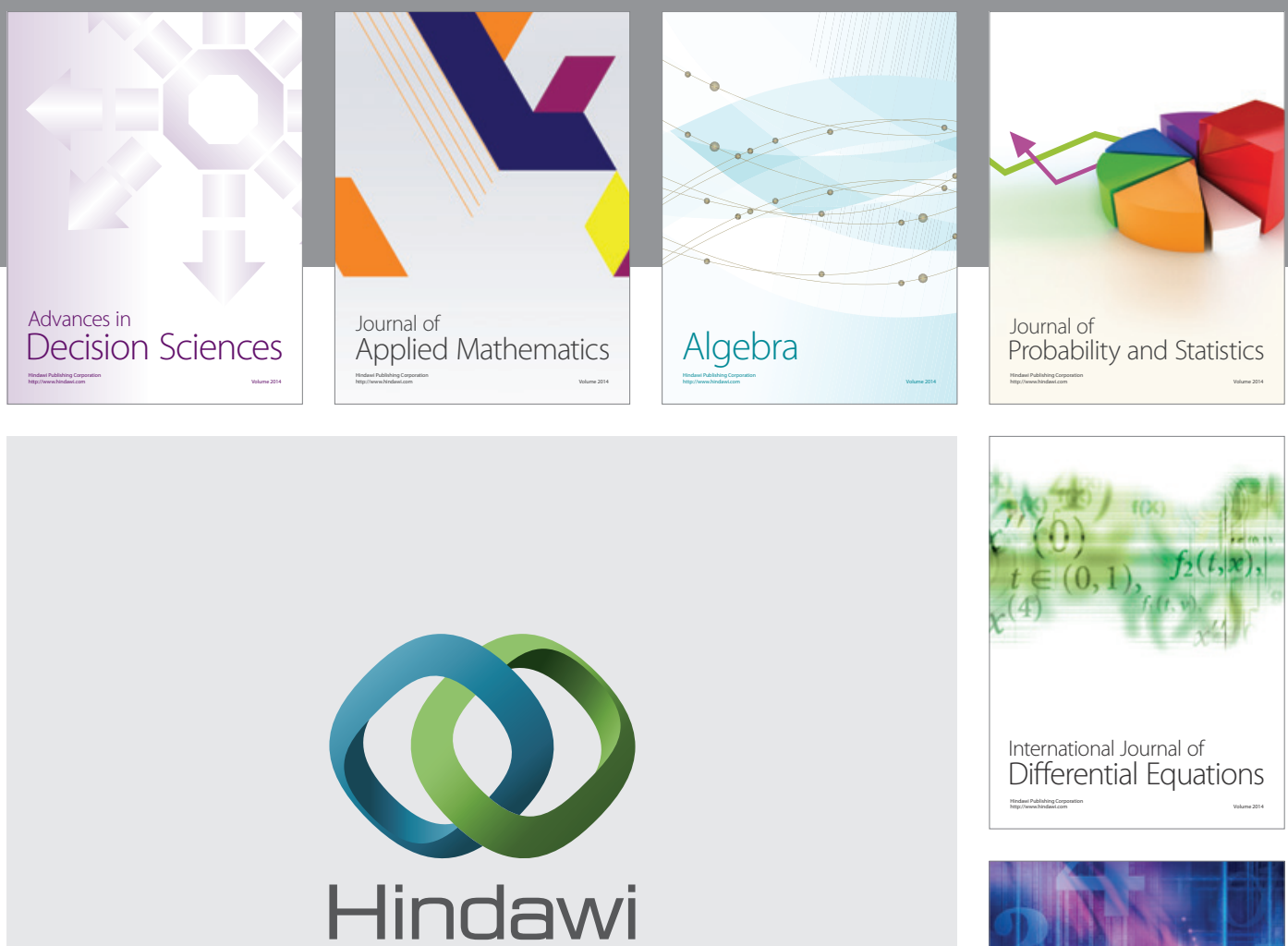

Submit your manuscripts at http://www.hindawi.com
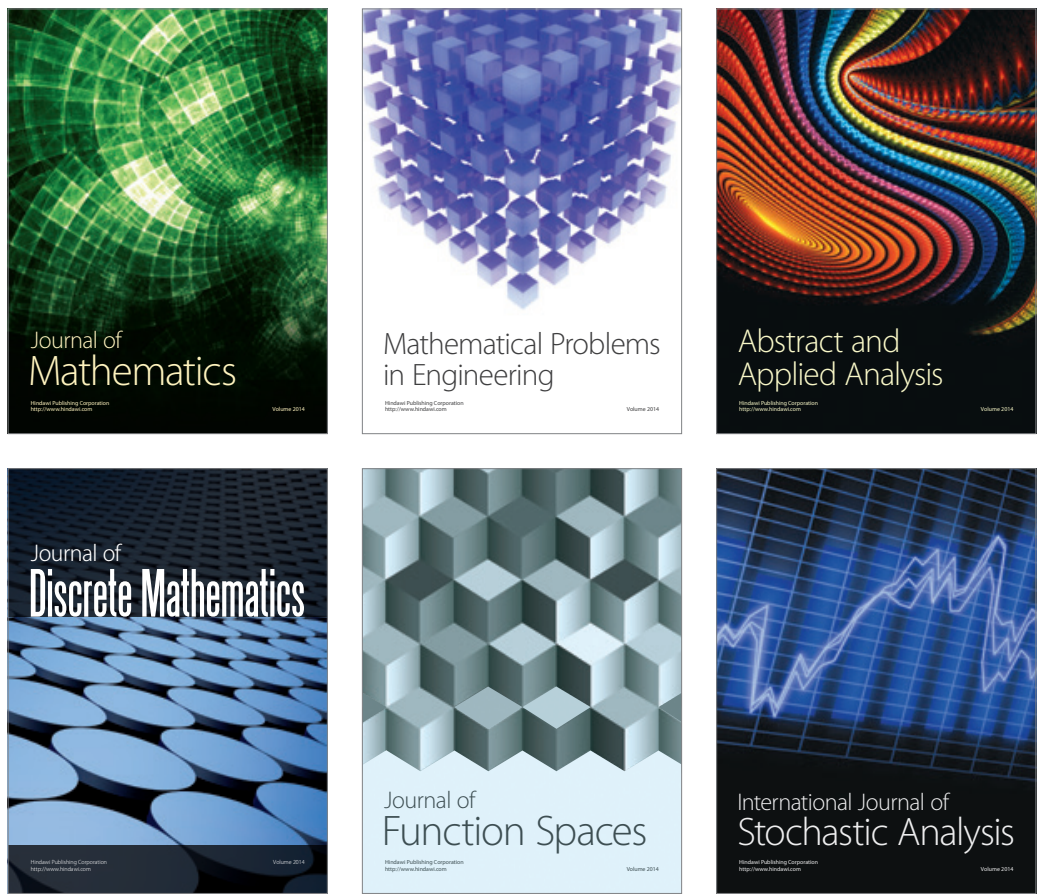

Journal of

Function Spaces

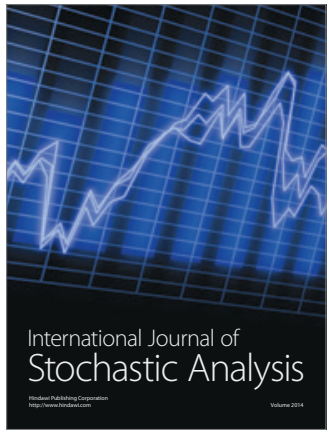

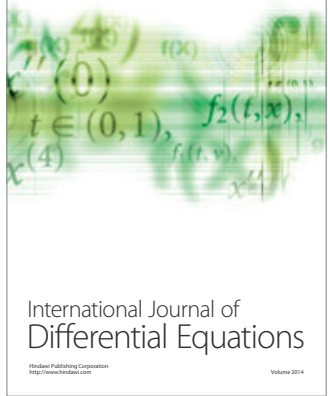
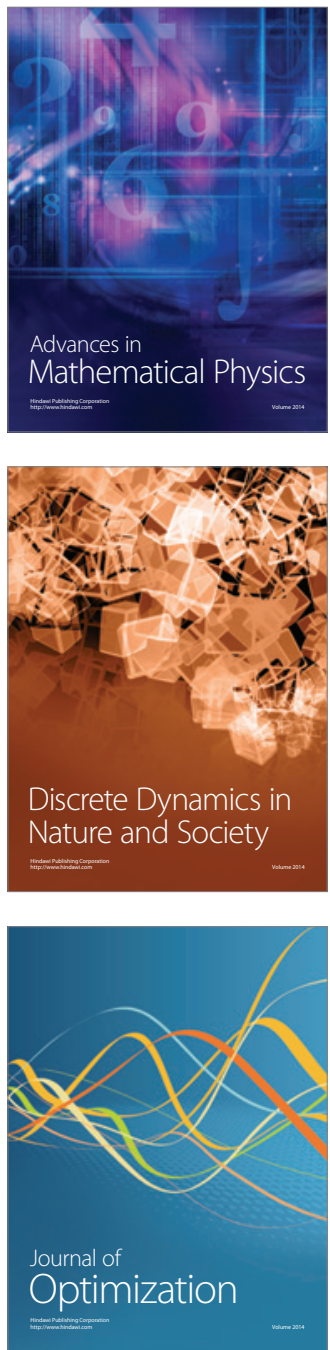